\title{
Correction to: Translation of the Gothenburg Trismus Questionnaire-2 into Telugu and its Validation for use in Indian Patients
}

\author{
Bylapudi Bhanu Prakash ${ }^{1}$. Sravankumar Chava ${ }^{1}$. Jonathan T. Gondi · L. M. Chandra Sekara Rao S ${ }^{1}$. \\ Caterina Finizia $^{2} \cdot$ T. Subramanyeshwar Rao ${ }^{3} \cdot$ Hemantkumar Onkar Nemade $^{1}$
}

(c) Indian Association of Surgical Oncology 2021/Published online: 12 July 2021

A Correction to this paper has been published: https://doi.org/10.1007/s13193-021-01384-8

\section{Correction to: Indian Journal of Surgical Oncology https://orcid.org/10.1007/s13193-021-01369-7}

The original version of this article inadvertently contained a mistake. Reason: authors incorrectly listed in PDF version while correctly presented in the html version (with Production). This is due to production template error. This is being corrected in this publication.

Publisher's Note Springer Nature remains neutral with regard to jurisdictional claims in published maps and institutional affiliations.
The original article can be found online at https://doi.org/10.1007/ s13193-021-01369-7

Hemantkumar Onkar Nemade

drhemantnemade@gmail.com

Bylapudi Bhanu Prakash

prakash.bylapudi@gmail.com

Sravankumar Chava

gmcsravan@gmail.com

Jonathan T. Gondi

jona314@gmail.com

L. M. Chandra Sekara Rao S

drlmcsraos@gmail.com

Caterina Finizia

caterina.finizia@orlss.gu.se
T. Subramanyeshwar Rao subramanyesh@gmail.com

1 Head and Neck Services, Department of Surgical Oncology, Basavatarakam Indo American Cancer Hospital and Research Institute, Hyderabad, India

2 Department of Otorhinolaryngology, Institute of Clinical Sciences, Sahlgrenska Academy at the University of Gothenburg, Sahlgrenska University Hospital, Gothenburg, Sweden

3 Department of Surgical Oncology, Basavatarakam Indo American Cancer Hospital and Research Institute, Hyderabad, India 